\title{
Pipe flow speed sensor based on Fiber Bragg Gratings
}

\author{
L. Rodriguez-Cobo*, M.A. Quintela, M. Lomer, A. Cobo, J.M. Lopez-Higuera \\ Photonics Engineering Group, University of Cantabria, Av los Castros S/N, 39005, \\ Santander, Spain
}

\begin{abstract}
A fluid flow speed sensor based on Fiber Bragg Gratings (FBGs) is proposed and demonstrated. Two FBGs are attached to an arc-shaped plastic piece at the same distance from both edges. The fluid flow to be measured pushes the structure stretching one side and compressing the other one. The sensor has been successfully checked by subjecting the device, to different air flow controlled conditions inside of a pipe. Low energy impact detection has been also reported with the proposed device.
\end{abstract}

Keywords: Fiber Bragg Grating, flow sensor, pipe, impact detection

\section{INTRODUCTION}

The global modern economy relies on an extensive network of pipelines which carries very different materials like industrial waste or raw materials such as gas, crude oil or even water. A pipeline network failure can provoke enormous economic and environmental losses and it can be prevented with the right maintenance. There are a lot of typical damages which can spoil a pipe such as corrosion due to de environmental conditions, excessive flow or pressure, transportation of corrosive waste, impacts of dragged materials, etc... Some of these parameters have been measured with different optical fiber technologies such as Brillouin distributed sensors applied to evaluate pipe deformation [1] or even to detect leakages.

Optical fiber sensors [2] have also been reported to measure flow speed. One example is the hot wire anemometry in which the cooling time of a heated wire (ie. optical fiber) is measured to determine the flow speed [3]. Hot wire anemometry is a much extended technique but usually it is oriented to measure very low flows and with a little density like air so, to apply this technique to harsher environments such as monitoring a waste pipe can be trickier. Other approach based on a deformable cantilever beam instrumented with a single Fiber Bragg Grating (FBG) have been also reported to measure wind speed in outdoor scenarios [4]. More dense fluid flow speed has been also measured by placing an optical fiber probe in the middle of a pipe [5]. With this technique based on index refraction, to detect different materials such as air bubbles in the middle of water has also been reported. The studied approaches show the feasibility of optical fiber sensors to measure flow speeds and detect anomalies inside the flow but usually with an impractical implementation for most of the harshest environments.

In this work, a FBG transducer designed to achieve a very good sensitivity to measure a fluid flow speed with a simpler installation procedure is shown. The ratio between sensitivity and fiber protection can be balanced to measure under very different conditions just by changing the holding material and dimensions. An arc shaped beam instrumented with FBGs is attached inside of a pipe to quantify the speed of the flowing fluid. The tested design was implemented to measure air flow speed for being the hardest fluid to be measured in sensitivity terms (lowest density). Some small objects were added to the air flow to hit the sensor in order to detect dragged elements.

\section{SENSOR ARCHITECTURE}

As shown in Figure1, the architecture of the fiber transducer is based on two FBGs symmetrically attached to the middle of an arc-shaped piece. The FBGs are attached to the piece in such a way that the fluid flux stretch the first FBG (saw from the incoming fluid) and compress the second one.

*luis.rodrigurez@unican.es; phone: (+34) 942 200877; fax: (+34) 942 200877;

OFS2012 22nd International Conference on Optical Fiber Sensors, edited by

Yanbiao Liao, Wei Jin, David D. Sampson, Ryozo Yamauchi, Youngjoo Chung, Kentaro Nakamura, Yunjiang Rao, Proc. of SPIE Vol. 8421, 84214E · C 2012 SPIE · CCC code: 0277-786/12/\$18 · doi: 10.1117/12.970621 


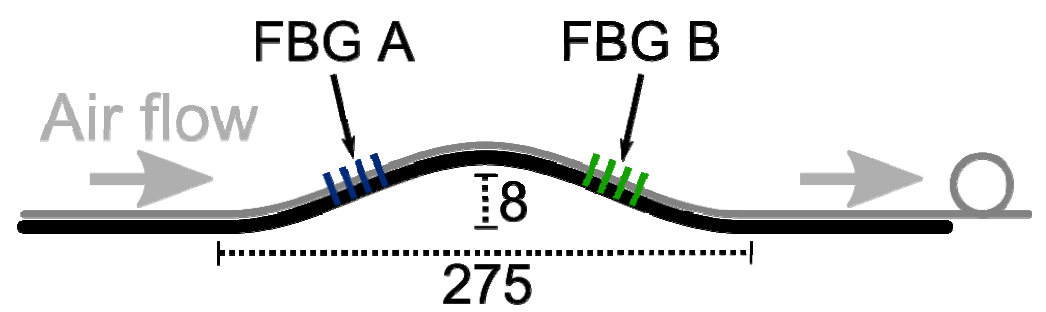

Figure 1. Flow speed sensor scheme. Two FBG transducers are attached symmetrically to the support arc piece. Dimensions are in millimeters

The sensitivity of the structure depends directly of the dimensions and materials employed as support. To measure the flow speed of a more dense fluid (for example crude oil), it is needed a stiff support material such as aluminum or even steel. In this paper, the fluid to be measured is air, so the chosen support material should be more elastic such as plastic. Concretely the employed plastic is Polyvinyl chloride (PVC) of $1 \mathrm{~mm}$ thickness and $25 \mathrm{~mm}$ of width in order to increase the surface in contact with the fluid. The two FBGs are bonded to the support plastic piece, at the same distance from the central point of the arc. After that a plastic cover is applied to the whole surface to protect the FBGs.

\section{EXPERIMENTAL SETUP}

The proposed design is attached to a plastic pipe of square section of $5 \mathrm{~cm}$ by $5 \mathrm{~cm}$. To the ending edge of the pipe, an anemometer is attached to measure the air speed. A compressed air tube is also attached to the initial edge with enough distance to the sensor to get a homogeneous air flow. By changing the pressure regulator the resulting air flow also changes.

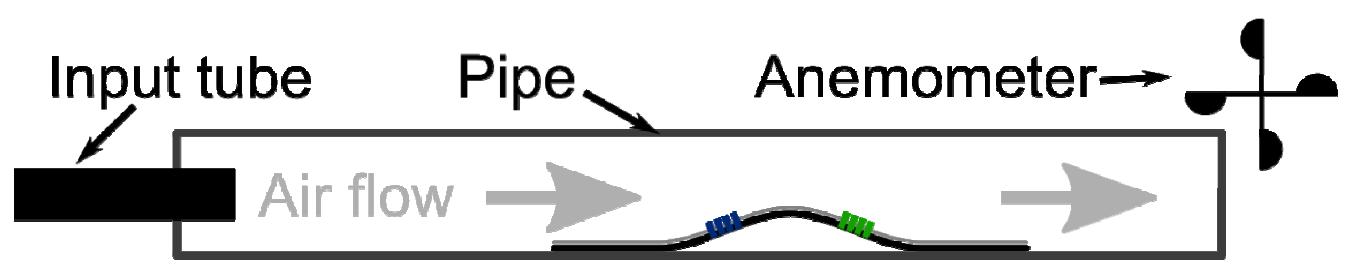

Figure 2. Experimental setup. The proposed sensor is installed inside a plastic pipe in which a controllable air flow has been created. An anemometer is also attached at the end of the pipe.

A seven steps sweep is made varying the generated air flow inside the pipe. The air flow is controlled by the pressure regulator and measured by the anemometer. The sweep is repeated changing the flow direction and modifying slightly the angular position of the sensor to quantify the influence of the position. FBGs are connected to an interrogation unit using a sampling frequency of $5 \mathrm{~Hz}$. In order to detect particles dragged by the flow some plastic and metallic pieces were introduced inside de air flow. For this purposes, FBGs are connected to the interrogation unit (Micron Si425-500) at higher sampling frequency $(250 \mathrm{~Hz})$ in order to get the hit response.

\section{RESULTS AND DISCUSSION}

Figure 3 shows the raw signals of the two FBGs during an air flow speed sweep from almost $1 \mathrm{~m} / \mathrm{s}$ down to $0 \mathrm{~m} / \mathrm{s}$ with 7 intermediate steps. The first FBG hit by the air flow (FBG_A) is stretched and the second one (FBG_B) is compressed. A possible imbalance of the two signals is usually caused by a non symmetrical placement or a non-homogeneous flow.

The obtained raw data is processed to get a single value. For this purpose, the average difference value between the two signals is computed. The taken window is of 40 samples ( 8 seconds) for each step. The obtained values from the FBG transducer are compared to the ones measured by the anemometer (Figure 4). 


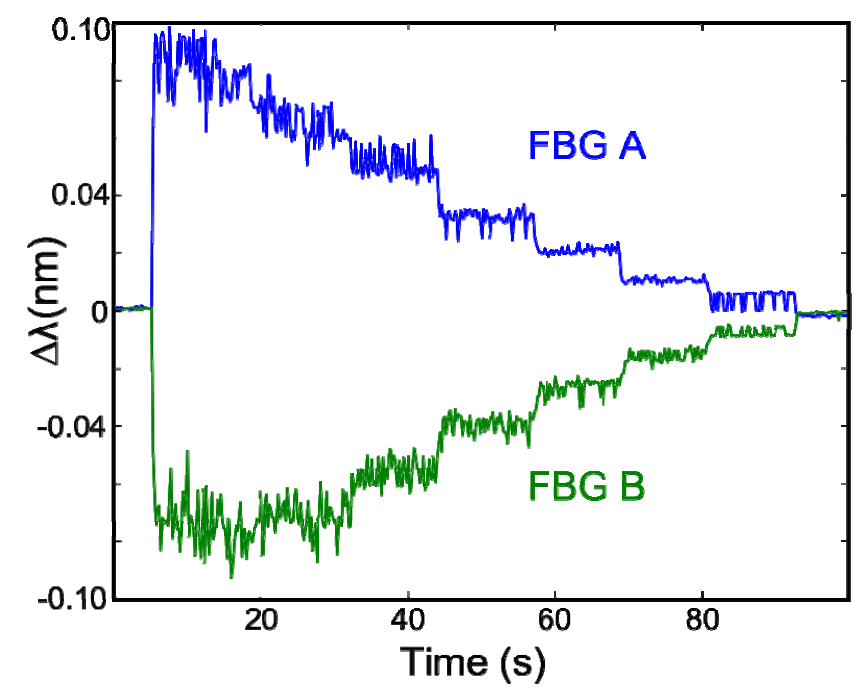

Figure 3. Raw signals from the two FBGs during an air flow speed sweep

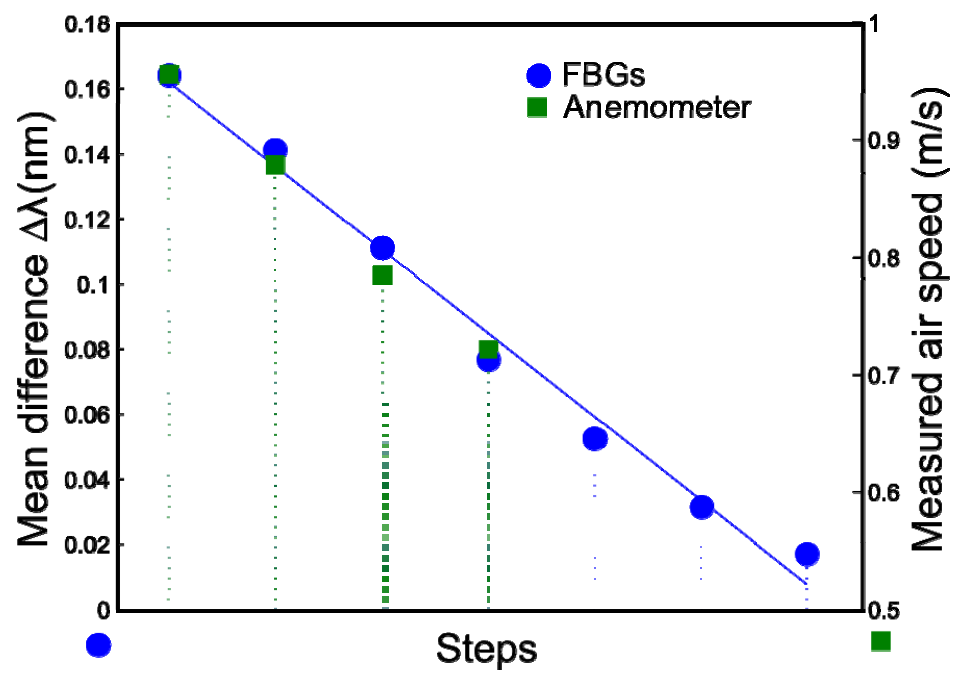

Figure 4. Processed signal form the FBG transducer compared with the measures of the anemometer

Due to the sensitivity of the employed anemometer just the 4 higher step values were quantified. The processed FBG data follows the same linear tend shown by the anemometer measures. If the angular position of the transducer varies with respect to the air flow, the same linear trend is maintained but with a different slope.

The FBG transducer is connected to a higher sampling frequency $(250 \mathrm{~Hz})$ to detect impacts of solid pieces dragged by the air flow. Two small pieces were dropped to hit the FBG transducer. The first piece was a plastic die of 0.8 grams and the second piece was a metallic nut of 1 gram. Both pieces are detected by the two FBG of the transducer. 


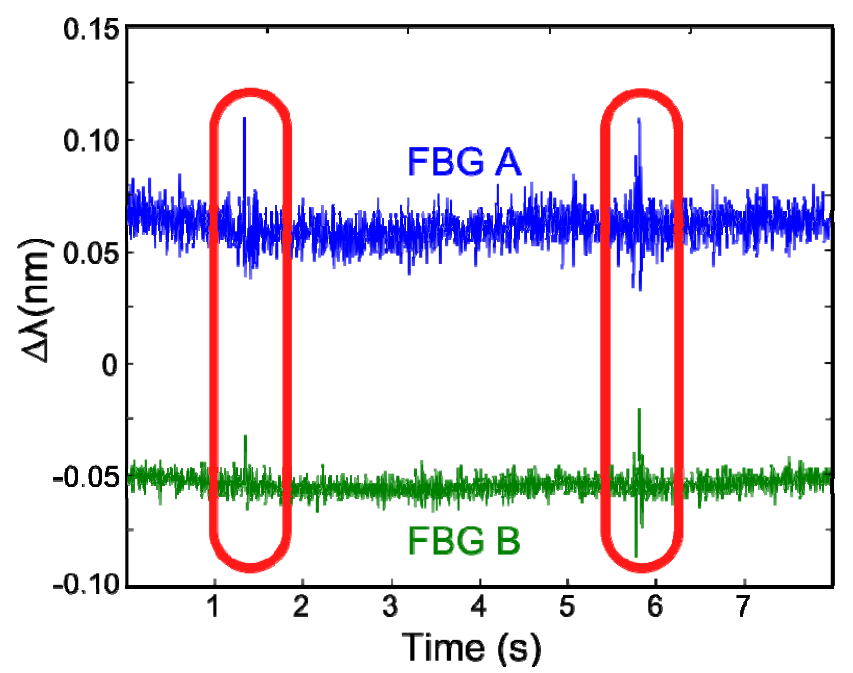

Figure 5. Raw signals from the FBGs of two solid pieces impacts at $250 \mathrm{~Hz}$.

\section{CONCLUSSION}

In this work a new flow speed sensor is tested. The arc-shaped transducer designed for its installation in pipes is subjected to a controllable air flow to quantify its optical response to different air flow speeds. The achieved results show a great agreement between the measured data and the provoked test conditions with an increased resolution over other techniques (anemometer). Despite the transducer was tested with air flow, with stiffer implementations more dense fluids can also be measured. In addition to the flow speed quantification, this transducer design is also capable of detecting low energy impacts coming from fluid dragged particles just by increasing the sampling frequency.

\section{ACKNOWLEDGMENTS}

This work has been supported by the project TEC2010-20224-C02-02 and grant AP2009-1403. Roberto Perez Sierra is also thanked for his valuable collaboration on the experimental stage.

\section{REFERENCES}

[1] Zou, L., Bao, X., Ravet, F., and Chen, L., "Distributed Brillouin fiber sensor for detecting pipeline buckling in an energy pipe under internal pressure," Applied optics, vol. 45, pp. 3372$3377,2006$.

[2] López-Higuera, J. M., Rodriguez Cobo, L., Quintela Incera, A., and Cobo, A., "Fiber optic sensors in structural health monitoring," Journal of Lightwave Technology, vol. 29, pp. 587-608, 2011.

[3] Cashdollar, L. J. and Chen, K. P., "Fiber Bragg grating flow sensors powered by in-fiber light," Sensors Journal, IEEE, vol. 5, pp. 1327-1331, 2005.

[4] Ma, G. M., Li, C. R., Jiang, J., Liang, J. Y., Luo, Y. T., and Cheng, Y. C., "A Passive Optical Fiber Anemometer for Wind Speed Measurement on High-Voltage Overhead Transmission Lines," Instrumentation and Measurement, IEEE Transactions on, pp. 1-6, 2012.

[5] Descamps, M., Oliemans, R. V. A., Ooms, G., and Mudde, R., "Air-water flow in a vertical pipe: experimental study of air bubbles in the vicinity of the wall," Experiments in Fluids, vol. 45, pp. 357-370, 2008. 